\title{
Indicadores de calidad del agua en lagunas insulares costeras con influencia turística: Cozumel e Isla Mujeres, Quintana Roo, México
}

\author{
Adrián Cervantes-Martínez* \\ Martha Angélica Gutiérrez-Aguirre \\ Universidad de Quintana Roo \\ Teresa Álvarez-Legorreta \\ El Colegio de la Frontera Sur
}

\section{Resumen}

A partir de variables físicas y químicas se analizó la calidad del agua para determinar la factibilidad del uso recreativo de dos lagunas costeras de la zona norte de Quintana Roo: laguna Colombia (LC), dentro del Parque Marino Nacional Arrecifes de Cozumel, y laguna Macax (LM), en el polígono del área natural protegida Costa Occidental de Isla Mujeres, Punta Cancún y Punta Nizuc. En ambas se realizan actividades de observación de flora y fauna, senderismo o incluso de puerto de abrigo; sin embargo, se desconoce su capacidad para efectuar actividades recreativas de contacto directo respetando las siguientes normatividades: a) criterios Ecológicos de Calidad del Agua CE-CCA-001/89; b) NOM-001-ECOL-1996, y c) criterios de aguas estuarinas y costeras de la Agencia de Protección Ambiental de Estados Unidos (Environmental Protection Agency, EPA). Se consideraron los cambios cíclicos naturales durante el año 2010, así como 12 indicadores de calidad (profundidad, transparencia, temperatura, salinidad, oxígeno disuelto, $\mathrm{pH}$, concentración de clorofila, demanda química y bioquímica de oxígeno, concentración de ortofosfatos, nitratos y nitritos). Las pruebas estadísticas mostraron que las lagunas no tienen características estáticas espacial ni temporalmente. De manera particular, sobrepasan los límites máximos de concentración de nutrimentos, según los criterios ecológicos para la protección de la vida acuática marina y la Nom-001; además, la calidad del agua puede clasificarse como pobre según los criterios de la epa. Los resultados servirán para planificar el uso de estos sistemas en función de sus características básicas.

\section{Palabras clave}

Área natural protegida, islas, turismo, lagunas costeras.

\section{*Correo electrónico: adcervantes@uqroo.mx}




\title{
Water quality indicators of coastal lagoons with toursim influence: Cozumel and Isla Mujeres, Quintana Roo, México
}

\begin{abstract}
The feasibility of recreational use of Laguna Colombia (LC) within the National Marine Park "Arrecifes de Cozumel”, and Laguna Macax (LM), within the natural protected area of Costa Occidental in Quintana Roo was surveyed using physical and chemical indicators of water quality. Activities related to nature observation are currently carried out, but the feasibility to support direct contact recreational activities is unknown. In this study the natural cyclic changes (2010) were considered using the following criteria: a) Ecological criteria of water quality (ce-cca-001/89), b) NOM-001-semarnat-1996, and c) EPA Ecological criteria for estuarine and coastal waters. Twelve indicators of water quality were considered (depth, transparency, water temperature, salinity, dissolved oxygen, $\mathrm{pH}$, chlorophyll, chemistry and biochemistry oxygen demand, and concentration of orthophosphate, nitrate, and nitrite). Statistical tests showed that the lagoons do not have static temporal or spatial features and according to ecological criteria, they exceed the maximum permissible concentration of nutrients and thus the water quality can be classified as poor. Results will be useful in planning the use of these systems in terms of their basic features.
\end{abstract}

Adrián Cervantes-Martínez Martha Angélica Gutiérrez-Aguirre Universidad de Quintana Roo Teresa Álvarez-Legorreta El Colegio de la Frontera Sur

\section{KeY WORDS}

Islands, protected natural area, tourism, coastal lagoons.

E-mail:adcervantes@uqroo.mx 


\section{Introducción}

La dimensión ambiental se ha explorado ampliamente en sistemas costeros de todo el mundo, considerando aspectos ecológicos, geológicos, oceanográficos y sociales (crecimiento poblacional, asentamientos humanos y turismo, entre otros). Alrededor de $60 \%$ de la población mundial se ha establecido en las zonas costeras (Aranda Cirerol, 2004), aunque estas representan un porcentaje muy pequeño del territorio disponible para habitar. En México, este fenómeno se ha agudizado debido al creciente interés por construir complejos turísticos y al apoyo para el desarrollo de turismo masivo. Esto se ha observado en particular en la región conocida como Caribe mexicano, la cual, de igual modo que el resto de las costas mexicanas, se ha poblado a una tasa más alta en comparación con el promedio nacional; por ejemplo, en Quintana Roo, la población ha crecido 350 \% en los últimos 20 años (Azuz-Adeath y Rivera-Arriaga, 2004). Todos estos habitantes demandan agua, ya sea para consumo humano o para fines recreativos, $y$, al margen del uso que se le dé, es necesario conocer la calidad de la misma.

El tipo de roca predominante en la región (porosa y altamente permeable) hace que sus sistemas acuáticos sean muy vulnerables a la contaminación, problema que en la Península de Yucatán es de carácter local y se asocia a los núcleos de desarrollo urbano, turístico y rural (Aguilar, 2003). Uno de los usos del agua en la región es el recreativo, de ahí que su calidad en centros turísticos sea primordial para proteger tanto la salud de los usuarios como de los organismos que en ella habitan. En la mayor parte de las zonas costeras de México existen factores que afectan la calidad del agua, como drenajes pluviales, descargas de aguas residuales tratadas, asentamientos irregulares que no cuentan con infraestructura de saneamiento y alcantarillado, embarcaciones y actividades de comercio informal, por lo cual resulta importante realizar estudios de la calidad de su agua, y evaluar su posible uso de acuerdo con las características que albergan.

El presente estudio pretende determinar la factibilidad del uso recreativo de contacto directo de dos lagunas costeras que se encuentran en el área de mayor afluencia turística de la zona norte de Quintana Roo: la laguna Colombia (LC) en Cozumel y la laguna Macax (LM) en Isla Mujeres, a partir de la identificación 
de indicadores físicos y químicos del agua (profundidad, transparencia, temperatura del agua, salinidad, oxígeno disuelto, pH, concentración de clorofila, demanda química y bioquímica de oxígeno -DQO y DBO- y concentración de ortofosfatos, nitratos y nitritos). Los resultados se contrastan con normas ambientales nacionales e internacionales, como la Nom-001-EcoL-1996 (Semarnat, 1997) los Criterios Ecológicos de Calidad del Agua CE-CCA-001/89 (Sedue, 1989) y los criterios de calidad de aguas estuarinas y costeras de la Agencia de Protección Ambiental de Estados Unidos (Environmental Protection Agency [EPA], 2004).

Lo anterior permitió hacer una propuesta de zonificación de las lagunas en función de sus características, así como detectar los indicadores que más información aportan para facilitar su manejo, uso y conservación. En la actualidad, en la sc se desarrollan actividades de observación de flora y fauna y senderismo, mientras que la Lm se usa básicamente como puerto de abrigo.

\section{Antecedentes}

Las lagunas costeras son un rasgo fisiográfico principal de las costas de la Península de Yucatán, se distinguen por su alta productividad, atractivo escénico y biodiversidad (Sandoval-Rojo et al., 1988; Herrera-Silveira, 2006). Se consideran ambientes delicados porque sus condiciones ambientales pueden ser transformadas a causa de cambios de usos de suelo, modificaciones hidrológicas y descarga de aguas residuales en las zonas con perturbación humana.

Los trabajos en ambientes costeros de la Península y con afluencia turística se han realizado sobre todo en la región norte, noreste (en específico en Holbox, Bojórquez y Nichupté) y centro-este (en particular en Bahía de la Ascensión) (Tran et al., 2002; Herrera-Silveira, 2006). Para Herrera-Silveira (2006), la importancia de estos sistemas se debe a que en ellos se desarrollan diferentes actividades impulsadas por su productividad, atractivo escénico y biodiversidad. Empero, los servicios ambientales de algunas de ellas se han reducido a consecuencia del incremento de descargas de aguas residuales, modificaciones hidrológicas y cambios de uso de la tierra.

Reyes y Merino (1991) reportaron que las actividades turísticas y las descargas no controladas de residuos cloacales ocasionadas por el desarrollo desmedido de 
Cancún, así como el dragado de más de $20 \%$ de su fondo original, repercutieron en la eutrofización de la laguna de Bojórquez. Las actividades humanas que se llevan a cabo en el área de influencia (incluidas las de recreación y desarrollo turístico) afectan de manera significativa el estado de salud de los sistemas acuáticos, y por ello es necesario monitorear su calidad.

Herrera-Silveira, Ramírez-Ramírez y Zaldivar (1998) trabajaron en lagunas costeras de la Península de Yucatán y propusieron que la calidad del agua se determina al identificar una serie de características asociadas con los contaminantes o con los procesos que sirven como indicadores para calificarla; así es posible establecer los valores máximos permisibles que presionan la vida acuática o que permiten al ser humano emplear el agua para su uso, cualquiera que este sea (recreativo, agrícola, industrial, consumo o contacto directo).

Herrera-Silveira, Comín y Capurro (2004) han hecho hincapié en conocer la función clave de los ecosistemas acuáticos, a partir de sus características básicas, para impulsar proyectos de desarrollo que tengan como objetivo principal la sustentabilidad en estos sistemas acuáticos, lo cual permitiría su aprovechamiento y su conservación.

En cuanto a los sistemas aquí analizados es pertinente mencionar que la región natural denominada laguna Colombia está ubicada al sur de la isla de Cozumel y en vista de la relevancia de los ecosistemas que posee, el 15 de julio de 1996 se decretó como área natural protegida, con la categoría de zona sujeta a conservación ecológica, refugio estatal de flora y fauna.

La laguna Macax se encuentra en Isla Mujeres, colinda al oeste con el Parque Marino Nacional Costa Occidental de Isla Mujeres, Punta Cancún y Punta Nizuc, y sirve de resguardo de embarcaciones de pequeño calado dedicadas a la pesca comercial, deportiva y esparcimiento en general.

\section{Metodología}

Para conocer la calidad del agua albergada en cada laguna se colectaron muestras considerando las épocas de secas (mayo de 2010) y de lluvias (octubre de 2010). 


\section{Trabajo de campo}

Se establecieron 18 sitios de muestreo en las dos lagunas costeras, diez en la laguna Colombia y ocho en la Macax (figuras 1 y 2). Los criterios para seleccionar los sitios fueron 1) complejidad del sistema, 2) ubicación respecto a la línea de costa y 3) dimensión de la laguna.

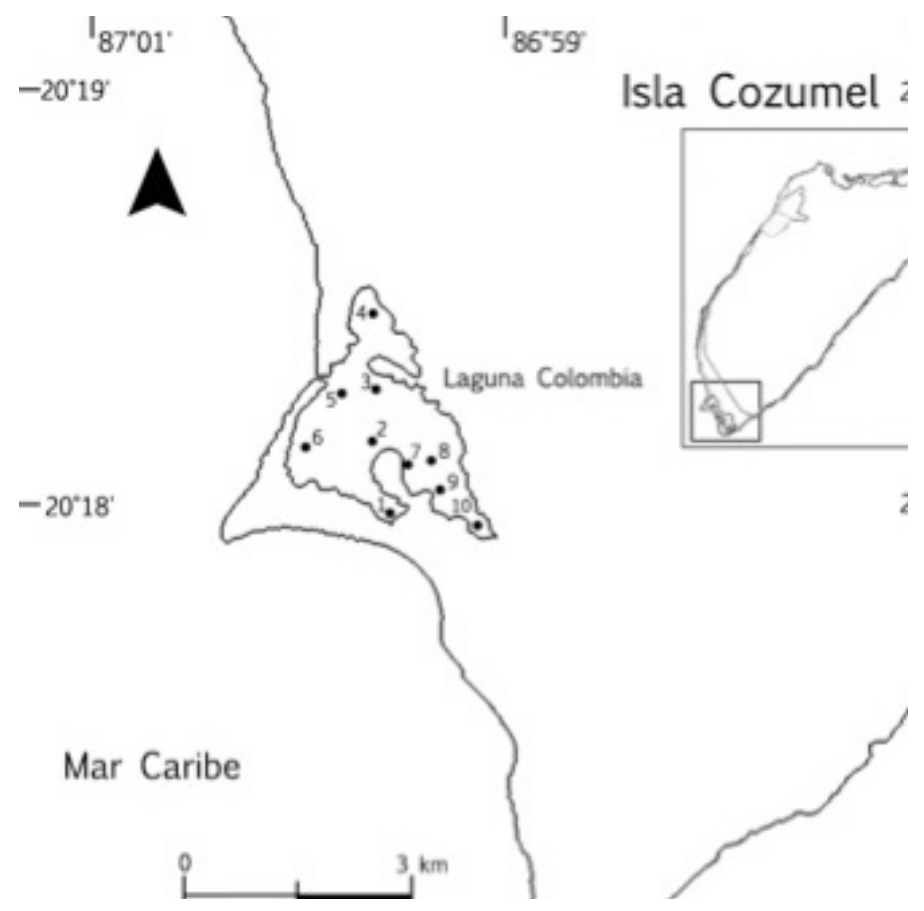

Figura 1. Estaciones de colecta consideradas en la laguna Colombia (épocas de secas y de lluvias, 2010) 


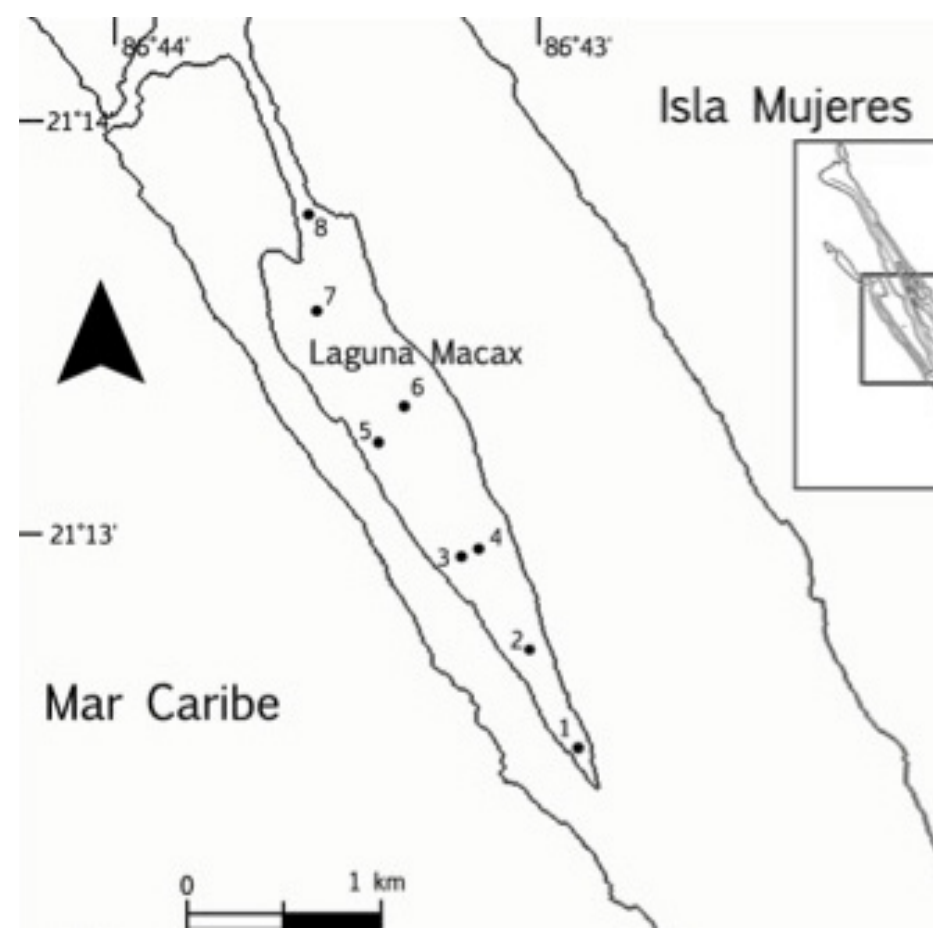

Figura 2. Estaciones de colecta consideradas en la laguna Macax (épocas de secas y de lluvias, 2010)

Durante las dos épocas climáticas de estudio se colectaron in situ 504 muestras de agua; 280 en la laguna Colombia y 224 en la Macax. Se determinaron la profundidad (m) y transparencia (m) con un disco de Secchi. La temperatura del agua $\left({ }^{\circ} \mathrm{C}\right)$, salinidad (ups), conductividad $\left(\mathrm{mS} \mathrm{cm}^{-1}\right)$, oxígeno disuelto (mg $\left.\mathrm{L}^{-1}\right)$ y $\mathrm{pH}$ se establecieron con una sonda multiparamétrica digital Hydrolab DataSonde 5.

También se colectaron muestras para conocer la concentración de nutrimentos inorgánicos (ortofosfatos $\left[\mathrm{PO}_{4}^{-}\right.$, nitratos $\left[\mathrm{NO}_{3}^{-}\right]$, nitritos $\left[\mathrm{NO}_{2}^{-}\right]$y silicatos $\left.\left[\mathrm{SiO}^{4}\right]\right)$, clorofila a $\left(\mathrm{mg} \mathrm{cm}^{-3}\right)$, DQO $\left(\mathrm{mg} \mathrm{L}^{-1}\right)$ y $\mathrm{DBO}_{5}\left(\mathrm{mg} \mathrm{L}^{-1}\right)$. Todas las muestras se conservaron en congelación para su posterior análisis en laboratorio. Todas las variables contaron con una muestra y dos réplicas. 


\section{Trabajo de laboratorio}

Los métodos analíticos empleados en la determinación de los nutrimentos se basaron en Parsons, Maita y Lalli (1984), en las normas oficiales mexicanas (DGN-SE, 2001a y 2001b) y en técnicas colorimétricas con la ayuda de un espectrofotómetro Thermo Spectronic Genesys 20.

La concentración de clorofila a se obtuvo siguiendo a APHA (1986); para conocer la DQo se utilizó la metodología de la NMX-AA-030-SCFI-2001 (DGN-SE, 2001b) y para la $\mathrm{DBO}_{5}$ la descrita en la NMX-AA-028- SCFI-2001 (DGN-SE, 2001a).

\section{Zonificación de cada laguna}

Para proponer áreas de monitoreo de calidad del agua en función de semejanzas o discontinuidades entre los sitios analizados, se realizaron análisis de agrupamiento uPGMA (Unweighted Pair Group Method with Arithmetic Mean), con distancias euclidianas. Debido a que las variables indicadoras de calidad del agua son de diferentes magnitudes, los datos se transformaron a logaritmo (excepto el pH) y el promedio de cada variable por punto de colecta se usó para los análisis de agrupamiento y la generación de dendrogramas con ayuda del software Multivariate Statistical Package (Mvsp versión 3.2).

\section{Indicadores de calidad del agua}

Para averiguar la contribución de cada variable sobre el comportamiento de los sistemas analizados, considerando la independencia de los registros obtenidos durante las secas y las lluvias, se realizó un análisis de componentes principales (ACP) con ayuda del mvsp 3.2.

Una vez terminados los análisis, para establecer la calidad del agua de ambas lagunas, los resultados se contrastaron con los Criterios Ecológicos de Calidad del Agua CE-CCA-001/89 y los indicadores para evaluación expuestos por la Comisión Nacional del Agua en la Nom-001-ECoL-1996, los cuales fijan los límites máximos permisibles de contaminantes y de indicadores de calidad para aguas nacionales de uso recreativo o para la protección de la vida acuática marina.

Por otro lado, con los criterios de la EPA fue posible precisar un índice de calidad del agua (Water Quality Index, weI) por sitio en tres rangos: buena, regular 
y pobre. El wQI es un indicador de la calidad de las aguas estuarinas y costeras propuesto por la Agencia de Protección Ambiental de Estados Unidos; se fundamenta en la información que aportan cinco variables: la concentración de nitrógeno, fósforo, clorofila a, oxígeno disuelto y la claridad del agua (EPA, 2004).

Por último, el índice trix es un indicador del nivel trófico de sistemas acuáticos costeros en función de cuatro variables: concentración de clorofila a nitrógeno total, fósforo total y oxígeno disuelto. Genera valores del 0 al 10; cuando estos oscilan de 0 a 2.5, la laguna costera se clasifica como oligotrófica; si van de 2.5 a 5.0 el sistema es mesotrófico; de 5 a 7.5, es eutrófico, y de 7.5 a 10, hipertrófico (Vollenweider et al., 1998).

En este estudio se propone una modificación del cálculo del índice trófico, considerando la suma de la concentración de nitratos y nitritos como indicador de nitrógeno total, y las determinaciones de ortofosfatos como indicador de fósforo para calcular el TRIx en cada sitio de colecta y por estaciones climáticas.

\section{Resultados}

\section{Zonificación de la laguna Colombia}

\section{SECAS}

El dendrograma resultante agrupa tres regiones espacialmente distintas de acuerdo con sus características físicas y químicas durante las secas (figura 3a). La primera es la región alrededor del punto 10, donde se registraron concentraciones elevadas de silicatos $\left(37.5 \mu \mathrm{M} \mathrm{L}^{-1}\right)$, baja $\mathrm{DBO}_{5}$ ( 0.6 a $\left.0.8 \mathrm{mg} \mathrm{L}^{-1}\right)$, baja DQo (2.0 a $\left.2.2 \mathrm{mg} \mathrm{L}^{-1}\right)$ y baja concentración de ortofosfatos $\left(0.007-0.08 \mu \mathrm{M} \mathrm{L}^{-1}\right)$ y de nitratos $\left(0.74 \mu \mathrm{M} \mathrm{L}^{-1}\right)$, así como una concentración de clorofila oscilante entre 0.19 y $0.27 \mathrm{mg} \mathrm{L}^{-1}$.

La segunda región comprendió las estaciones 5 y 6, porque en ellas se registraron las concentraciones de silicatos y nitratos más bajas durante las secas (2.4-2.6 $\mu \mathrm{M} \mathrm{L}^{-1}$ y 0.17-0.32 $\mu \mathrm{M} \mathrm{L}^{-1}$ ), con una tendencia de mayor $\mathrm{DBO}_{5}$ (5.1-5.6 $\left.m g \mathrm{~L}^{-1}\right)$, una concentración intermedia de ortofosfatos $\left(0.38-0.4308 \mu \mathrm{M} \mathrm{L}^{-1}\right)$ y una elevada DQo (10.1-11.1 $\left.\mathrm{mg} \mathrm{L}^{-1}\right)$ en comparación con el resto de la laguna.

En la tercera región (estaciones 1-4, 7-9) se hallaron concentraciones intermedias de silicatos (3.6-7.5 $\left.\mu \mathrm{M} \mathrm{L}^{-1}\right)$, una $\mathrm{DBO}_{5}$ intermedia (2.0-5.6 $\left.\mathrm{mg} \mathrm{L}^{-1}\right)$, una concentración de ortofosfatos entre 0.33 y $0.56 \mu \mathrm{M} \mathrm{L}^{-1}$; una DQo intermedia 
(4.0 a $8.3 \mathrm{mg} \mathrm{L}^{-1}$ ) y la concentración de nitratos más alta en comparación con el resto del sistema (1.3-2.37 $\left.\mu \mathrm{M} \mathrm{L}^{-1}\right)$. Aquí, durante las secas, la concentración de clorofila osciló entre 0.09 y $0.67 \mathrm{mg} \mathrm{L}^{-1}$ en promedio.

\section{LLUVIAS}

El análisis de agrupamiento sugiere que la regionalización espacial de la laguna es poco clara durante las lluvias (figura $3 \mathrm{~b}$ ). En el grupo formado por las estaciones 5 a 9 se registraron concentraciones de nitratos de 1.38 a $1.94 \mu \mathrm{M} \mathrm{L}^{-1}$, ortofosfatos de 0.38 a $0.48 \mu \mathrm{M} \mathrm{L}^{-1}, \mathrm{DBO}_{5}$ de 2.0 a 5-66 $\mathrm{mg} \mathrm{L}^{-1}$, DQO de 4.0 a 11.13 $\mathrm{mg} \mathrm{L}^{-1}$ y clorofila de 0.28 a $1.4 \mathrm{mg} \mathrm{L}^{-1}$.

En el grupo integrado por las estaciones 3 y 10 (figura 3b) las concentraciones de nitratos (0.74-1.65 $\left.\mu \mathrm{M} \mathrm{L}^{-1}\right)$, ortofosfatos $\left(0.05-0.43 \mu \mathrm{M} \mathrm{L}^{-1}\right)$, clorofila (0.37-0.67 $\left.\mathrm{mg} \mathrm{L}^{-1}\right)$, дво (0.66-4.73 $\left.\mathrm{mg} \mathrm{L}^{-1}\right)$ у DQо $\left(2.0-7.53 \mathrm{mg} \mathrm{L}^{-1}\right)$ tendieron a ser menores que las del grupo anterior. Las estaciones 1,2 y 4 constituyeron puntos aislados (figura 3b), donde las variables se manifestaron como de transición entre los dos grupos anteriores.
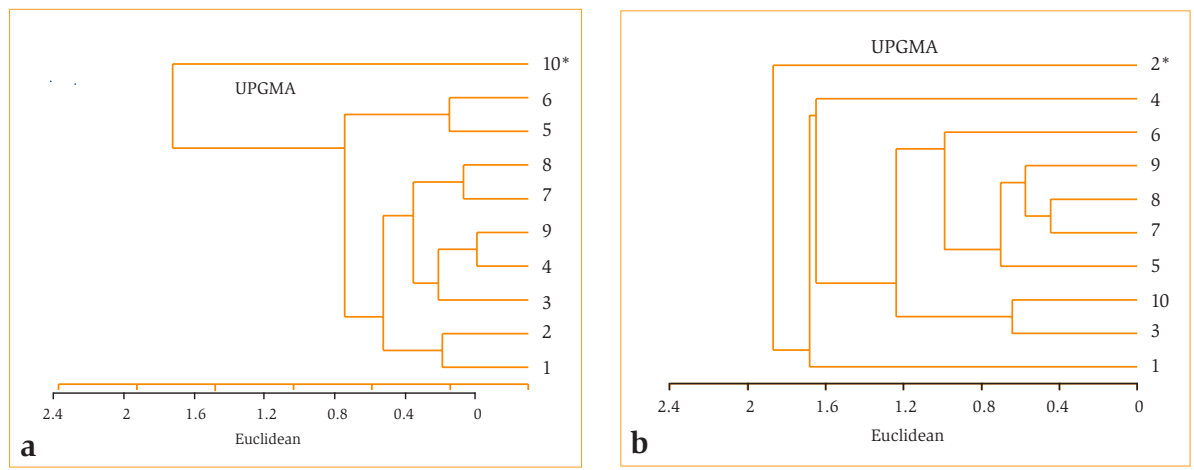

* Nota: Los números al extremo derecho de cada dendrograma corresponden a las estaciones de colecta mostradas en la figura 1.

Figura 3. Dendrograma de distancias entre las estaciones de colecta en la laguna Colombia durante las épocas de secas (a) y de lluvias (b). 


\section{Zonificación de la laguna Macax}

\section{SECAS}

De acuerdo con el dendrograma (figura 4a), durante las secas se registró un incremento gradual en la $\mathrm{DBO}_{5}$ (de 0.26 hasta $1.2 \mathrm{mg} \mathrm{L}^{-1}$ ), la concentración de clorofila (de 0.07 a $0.2 \mathrm{mg} \mathrm{L}^{-1}$ ), el pH (de 7.7 a 8.2 ) y en la concentración de oxígeno disuelto (de 2.5 a $7.6 \mathrm{mg} \mathrm{L}^{-1}$ ) de las regiones sureste a noreste de la laguna. En el mismo sentido se observó un decremento gradual en la concentración de nitritos (1.13 a $\left.0.23 \mu \mathrm{M} \mathrm{L}^{-1}\right)$, silicatos (5.42 a $\left.1.7 \mu \mathrm{M} \mathrm{L}^{-1}\right)$ y nitratos (de 2.1 a $0 \mu \mathrm{M} \mathrm{L}^{-1}$ ).

\section{LLUVIAS}

El comportamiento fue semejante al descrito; se observó una disminución gradual de sureste a noroeste en la concentración de nitritos y nitratos. Sin embargo, durante las lluvias se encontraron discontinuidades en dos regiones de la laguna (figura 4b) debido a la concentración de ortofosfatos, que varió entre 0.8 y $0.2 \mu \mathrm{M} \mathrm{L}^{-1}$ en las estaciones 1 y 2 y se mantuvo constante $\left(0.007 \mu \mathrm{M} \mathrm{L}^{-1}\right)$ en las estaciones 3 a 8 .

Por otro lado, la concentración de clorofila fue mayor en las estaciones 1 y 2 (0.28-0.33 $\left.\mathrm{mg} \mathrm{L}^{-1}\right)$, en comparación con la de las estaciones 3 a 8 (entre 0.07 y $\left.0.2 \mathrm{mg} \mathrm{L}^{-1}\right)$.
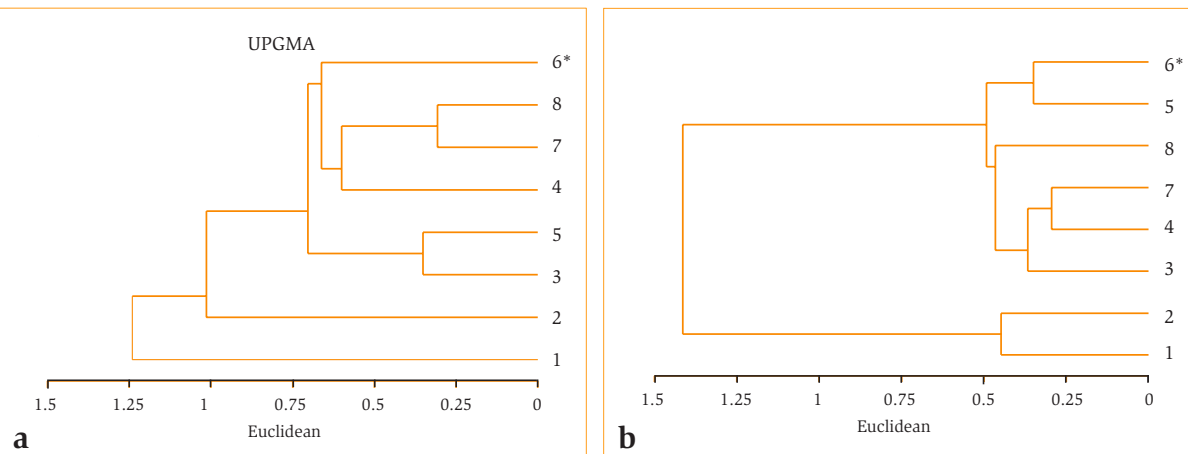

* Nota: Los números al extremo derecho de cada dendrograma corresponden a las estaciones de colecta mostradas en la figura 2.

Figura 4. Dendrograma de distancias entre estaciones de colecta en la laguna Macax durante las épocas de secas (a) y de lluvias (b). 


\section{Indicadores de calidad del agua}

\section{Laguna Colombia}

Durante los meses de secas, la mayor variabilidad en el sistema se explicó por los dos primeros ejes (82.5\%), y las cinco variables principales fueron la concentración de ortofosfatos, nitritos, nitratos, clorofila a y silicatos (cuadro 1).

Cuadro 1. Análisis de Componentes Principales (ACP) de variables analizadas en Laguna Colombia, durante secas.*

\begin{tabular}{lccc} 
& EJE 1 & EJE 2 & EJE 3 \\
Eigenvalores & 0.340 & 0.132 & 0.067 \\
Porcentaje de traza & 59.492 & 23.078 & 11.797 \\
Porcentaje acumulado & 59.492 & 82.570 & 94.367 \\
ConTRIBUCión DE LAS VARIABLES A LOS TRES PRIMEROS COMPONENTES (EJES 1 A 3 ) \\
\hline Oxígeno disuelto & -0.058 & 0.040 & 0.150 \\
Temperatura del agua & -0.007 & -0.001 & 0.021 \\
Salinidad & -0.025 & 0.002 & 0.023 \\
pH & 0.078 & -0.071 & -0.035 \\
Ortofosfatos & $\mathbf{0 . 4 4 4}$ & $\mathbf{- 0 . 2 3 7}$ & $\mathbf{0 . 1 0 9}$ \\
Nitritos & $\mathbf{0 . 2 4 4}$ & $\mathbf{- 0 . 5 6 3}$ & $\mathbf{0 . 5 9 2}$ \\
Nitratos & $\mathbf{0 . 1 7 5}$ & $\mathbf{- 0 . 1 7 4}$ & $\mathbf{0 . 2 1 1}$ \\
Silicatos & $\mathbf{0 . 5 2 9}$ & -0.081 & $\mathbf{0 . 3 2 3}$ \\
Clorofila a & $\mathbf{0 . 2 8 8}$ & $\mathbf{0 . 7 5 8}$ & $\mathbf{0 . 5 6 3}$ \\
Demanda bioquímica de oxígeno & $\mathbf{0 . 4 5 0}$ & -0.009 & $\mathbf{- 0 . 3 4 4}$ \\
Demanda química de oxígeno & $\mathbf{0 . 3 4 8}$ & 0.086 & $\mathbf{- 0 . 1 6 6}$ \\
Profundidad & $\mathbf{0 . 1 2 4}$ & -0.042 & $\mathbf{0 . 0 3 4}$ \\
\hline
\end{tabular}

* Las variables con mayor contribución en negritas. 
Durante las lluvias, los tres primeros ejes explicaron $89.4 \%$ de la variabilidad en el sistema, y la concentración de nitratos, ortofosfatos y DQo fueron las variables con mayor contribución a los tres primeros componentes (cuadro 2).

Cuadro 2. Análisis de Componentes Principales (ACP) de las variables analizadas en Laguna Colombia, durante Iluvias*

\begin{tabular}{lccc} 
& EJE 1 & EJE 2 & EJE 3 \\
Eigenvalores & 0.511 & 0.349 & 0.116 \\
Porcentaje de traza & 46.835 & 32.030 & 10.607 \\
Porcentaje acumulado & 46.835 & 78.865 & 89.472 \\
ConTRIBución DE LAS VARIABLES A LOS TRES PRIMEROS COMPONENTES (EJES 1 & A 3) \\
\hline Profundidad & 0.003 & 0.086 & -0.085 \\
Oxígeno disuelto & -0.029 & 0.090 & -0.060 \\
Temperatura del agua & -0.001 & 0.001 & 0.001 \\
Salinidad & -0.001 & -0.003 & 0.003 \\
pH & -0.050 & $\mathbf{0 . 1 5 0}$ & -0.039 \\
Ortofosfatos & $\mathbf{0 . 5 3 9}$ & $\mathbf{0 . 4 7 3}$ & $\mathbf{0 . 1 1 1}$ \\
Nitritos & 0.030 & -0.050 & $\mathbf{0 . 1 4 5}$ \\
Nitratos & $\mathbf{0 . 7 2 8}$ & $\mathbf{- 0 . 5 0 2}$ & $\mathbf{0 . 2 8 7}$ \\
Silicatos & 0.012 & $\mathbf{0 . 1 4 1}$ & $\mathbf{- 0 . 2 3 3}$ \\
Clorofila a & 0.064 & 0.019 & $\mathbf{- 0 . 4 1 3}$ \\
Demanda bioquímica de oxígeno & $\mathbf{- 0 . 3 5 6}$ & 0.065 & $\mathbf{0 . 7 8 9}$ \\
Demanda química de oxígeno & $\mathbf{0 . 2 1 0}$ & $\mathbf{0 . 6 7 7}$ & $\mathbf{0 . 1 5 8}$ \\
\hline
\end{tabular}

* Las variables con mayor contribución en negritas. 


\section{LaGuna MacaX}

El resultado sugiere que $87.5 \%$ de la variabilidad en el sistema se explicó por los tres primeros ejes y que las variables que contribuyeron a este comportamiento durante el tiempo de secas fueron la $\mathrm{DBO}_{5}$, la concentración de nitritos, silicatos, clorofila a y el pH (cuadro 3).

Cuadro 3. Análisis de Componentes Principales (PCA) de las variables analizadas en Laguna Macax, durante secas*

\begin{tabular}{|c|c|c|c|c|}
\hline & EJE 1 & EJE 2 & EJE 3 & EJE 4 \\
\hline Eigenvalores & 0.229 & 0.080 & 0.061 & 0.040 \\
\hline Porcentaje de traza & 54.120 & 18.946 & 14.516 & 9.393 \\
\hline Porcentaje acumulado & 54.120 & 73.066 & 87.582 & 96.759 \\
\hline \multicolumn{5}{|c|}{ CONTRIBUCIÓN DE LAS VARIABLES A LOS CUATRO PRIMEROS COMPONENTES (EJES 1 A 4) } \\
\hline Profundidad & -0.009 & -0.019 & -0.362 & 0.872 \\
\hline Oxígeno disuelto & 0.228 & -0.398 & -0.027 & -0.107 \\
\hline Temperatura del agua & -0.001 & -0.003 & 0.017 & -0.039 \\
\hline Salinidad & -0.006 & -0.002 & 0.014 & -0.019 \\
\hline $\mathrm{pH}$ & 0.301 & -0.121 & -0.295 & -0.106 \\
\hline Ortofosfatos & -0.061 & -0.070 & 0.128 & -0.179 \\
\hline \multicolumn{2}{|c|}{ 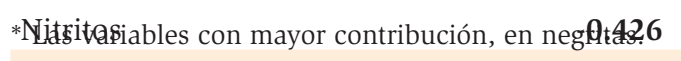 } & 0.144 & 0.369 & 0.053 \\
\hline Nitratos & -0.037 & 0.814 & -0.090 & -0.038 \\
\hline Silicatos & -0.157 & -0.299 & 0.311 & 0.231 \\
\hline Clorofila a & 0.406 & 0.105 & -0.333 & -0.182 \\
\hline Demanda bioquímica de oxígeno & 0.694 & 0.179 & 0.632 & 0.263 \\
\hline Demanda química de oxígeno & -0.008 & -0.080 & 0.113 & -0.151 \\
\hline
\end{tabular}


Dos ejes determinaron 91.9 \% de la variabilidad de la laguna Macax durante las lluvias, y las variables con mayor incidencia fueron la concentración de ortofosfatos y la clorofila a (cuadro 4).

Cuadro 4. Análisis de Componentes Principales (ACP) de las variables analizadas en Laguna Macax, durante Iluvias

\begin{tabular}{lcc} 
& EJE 1 & EJE 2 \\
Eigen valores & 0.422 & 0.035 \\
Porcentaje de traza & 84.955 & 6.963 \\
Porcentaje acumulado & 84.955 & 91.918 \\
\hline ConTrIBución DE LAS VARIABLES A Los Dos PRIMEROS COMPONENTES \\
(EJEs 1 A 2) & \\
\hline Profundidad & 0.026 & 0.130 \\
Oxígeno disuelto & -0.082 & 0.050 \\
Temperatura del agua & -0.002 & -0.003 \\
Salinidad & -0.003 & 0.002 \\
pH & -0.003 & 0.011 \\
Ortofosfatos & $\mathbf{0 . 8 9 7}$ & $-\mathbf{0 . 2 4 0}$ \\
Nitritos & $\mathbf{0 . 2 4 9}$ & -0.083 \\
Nitratos & $\mathbf{0 . 1 6 3}$ & 0.102 \\
Silicatos & 0.101 & -0.149 \\
Clorofila a & $\mathbf{0 . 2 7 6}$ & $\mathbf{0 . 9 2 1}$ \\
Demanda bioquímica de oxígeno & -0.110 & $\mathbf{0 . 1 8 5}$ \\
Demanda química de oxígeno & -0.040 & -0.036 \\
\hline
\end{tabular}

* Las variables con mayor contribución, en negritas.

\section{Índices de calidad del agua}

Laguna Colombia

- Oxígeno disuelto: en las secas la estación 1 y en las lluvias las estaciones 1, 3, 4 y 7 no presentaron el límite mínimo establecido en los criterios ecológicos para la protección de la vida acuática marina $\left(5 \mathrm{mg} \mathrm{L}^{-1}\right)$. 
- Demanda bioquímica de oxígeno: todos los valores registrados se encontraron debajo del límite máximo para explotación pesquera (150 mg $\left.\mathrm{L}^{-1}\right)$ y uso recreativo y estuarios $\left(75 \mathrm{mg} \mathrm{L}^{-1}\right)$ establecido en la Nom-001ECOL-1996.

- $p H$ : todos los valores se hallaron dentro del rango permitido en la Nom001-ECol-1996 para descargas de aguas residuales en aguas y bienes nacionales (5-10 unidades de $\mathrm{pH}$ ).

- Ortofosfatos, nitratos, nitritos: los promedios en todos los sitios de colecta y en ambas épocas climáticas fueron superiores a los límites máximos establecidos en los criterios ecológicos para la protección de la vida acuática marina $\left(0.002 \mu \mathrm{M} \mathrm{L}^{-1}, 0.04 \mu \mathrm{M} \mathrm{L}^{-1}\right.$ y $0.002 \mu \mathrm{M} \mathrm{L}^{-1}$, respectivamente).

- Temperatura: todos los valores permanecen dentro del rango permitido en la Nom-001-ECOL-1996 para descargas de aguas residuales en aguas y bienes nacionales $\left(40^{\circ} \mathrm{C}\right.$ de temperatura promedio diaria).

De acuerdo con los criterios de la EPA, la calidad del agua de cada sitio analizado en la laguna Colombia es pobre tanto en la época de secas como en la de lluvias, porque al menos dos o más indicadores se clasificaron de igual manera en cada sitio (cuadro 5).

Laguna MacaX

- Oxígeno disuelto: en las secas, la estación 1 fue la única que no registró el mínimo establecido en los criterios ecológicos, mientras que en las lluvias en ninguna estación de colecta se observó el mínimo requerido.

- Demanda bioquímica de oxígeno: todos los valores estaban debajo del límite máximo establecido en la Nom-001-ECoL-1996.

- $p H$ : todos los valores se ubicaron dentro del rango permitido en la Nom001-ECOL-1996.

- Ortofosfatos, nitritos, nitratos: en las dos temporadas de recolección, los promedios de las determinaciones de ortofosfatos y nitritos en todos los sitios de colecta fueron superiores a los límites máximos establecidos. En cuanto a los nitratos, en las secas solo se sobrepasa el límite en las 
Cuadro 5. Índice trófico (TRIX) e índice de Calidad del Agua de la EPA (wQI) por estación de colecta y temporada climática, determinado en lagunas Colombia y Macax*

\begin{tabular}{|c|c|c|c|c|}
\hline \multicolumn{5}{|c|}{ LAGUNA COLOMBIA } \\
\hline & \multicolumn{2}{|c|}{ TRIX } & \multicolumn{2}{|c|}{ WQI } \\
\hline & Secas & Lluvias & Secas & Lluvias \\
\hline 1 & 0.35 & 0.44 & Pobre & Pobre \\
\hline 2 & 0.44 & 0.36 & Pobre & Regular \\
\hline 3 & 0.69 & 0.65 & Pobre & Pobre \\
\hline 4 & 0.80 & 0.70 & Pobre & Pobre \\
\hline 5 & 0.78 & 0.62 & Pobre & Pobre \\
\hline 6 & 0.72 & 0.58 & Pobre & Pobre \\
\hline 7 & 0.72 & 0.41 & Pobre & Pobre \\
\hline 8 & 0.73 & 0.42 & Pobre & Pobre \\
\hline 9 & 0.82 & 0.6 & Pobre & Pobre \\
\hline 10 & 0.33 & 0.54 & Pobre & Pobre \\
\hline \multicolumn{5}{|c|}{ LAGUNA MACAX } \\
\hline & \multicolumn{2}{|c|}{ TRIX } & \multicolumn{2}{|c|}{ WQI } \\
\hline 1 & 0.46 & 1.1 & Pobre & Pobre \\
\hline 2 & 0.65 & 1.0 & Pobre & Pobre \\
\hline 3 & 0.68 & 0.7 & Pobre & Pobre \\
\hline 4 & 0.53 & 0.7 & Pobre & Pobre \\
\hline 5 & 0.44 & 0.9 & Pobre & Pobre \\
\hline 6 & 0.71 & 0.8 & Pobre & Pobre \\
\hline 7 & 0.61 & 0.7 & Pobre & Pobre \\
\hline 8 & 0.39 & 0.9 & Pobre & Pobre \\
\hline
\end{tabular}

*Los números de la primera columna corresponden a las estaciones de colecta mostradas en las figuras 1 y 2 . 
estaciones 1 y 2 ; mientras que en las lluvias las concentraciones fueron más altas que el límite en todas las estaciones.

- Temperatura: todos los registros reportaron valores dentro del rango permitido en la NOM-001-ECOL-1996.

Según los criterios de la EPA, la calidad del agua de cada sitio analizado en la laguna Macax es pobre tanto en las secas como en las lluvias, pues al menos dos o más indicadores se clasificaron de igual manera en cada sitio (cuadro 5).

\section{Índice trófico}

Las lagunas analizadas presentaron condiciones de oligotrofia en las dos temporadas (secas y lluvias), de acuerdo con los resultados del índice trófico TRIX (cuadro 5).

Durante las secas, en la laguna Colombia el índice osciló entre 0.35 y 0.82; y en las lluvias el rango varió de 0.36 a 0.70 . Por otro lado, en la laguna Macax, el TRIX osciló entre 0.44 y 0.71 en secas y de 0.7 a 1.1 en lluvias.

\section{Discusión}

A partir de las diferencias a escala espacial y temporal encontradas en los sistemas analizados, se presentan sugerencias de periodicidad, selección de sitios e indicadores de las condiciones de las lagunas Colombia y Macax en función de sus características (cuadro 6). Esta propuesta pretende facilitar el uso y manejo de los sistemas.

En cuanto al monitoreo espacial de las variables físicas y químicas de la laguna Colombia se proponen tres áreas durante la temporada de secas: la primera se ubica en la estación 10, que constituye un canal angosto de conexión entre la laguna Colombia y la laguna Chunchacaab; la segunda comprende la región oeste del sistema (estaciones 5 y 6), caracterizada por ser una región de conexión entre la laguna y el mar, a través de un canal angosto (20 a 30 m de ancho) (figura 1), y la tercera va desde la región centro hacia el este del sistema (estaciones 1-4, 7-9). 
Cuadro 6. Sitios recomendables para monitoreo de indicadores de calidad del agua de las Lagunas Colombia y Macax en función de la temporada climática*

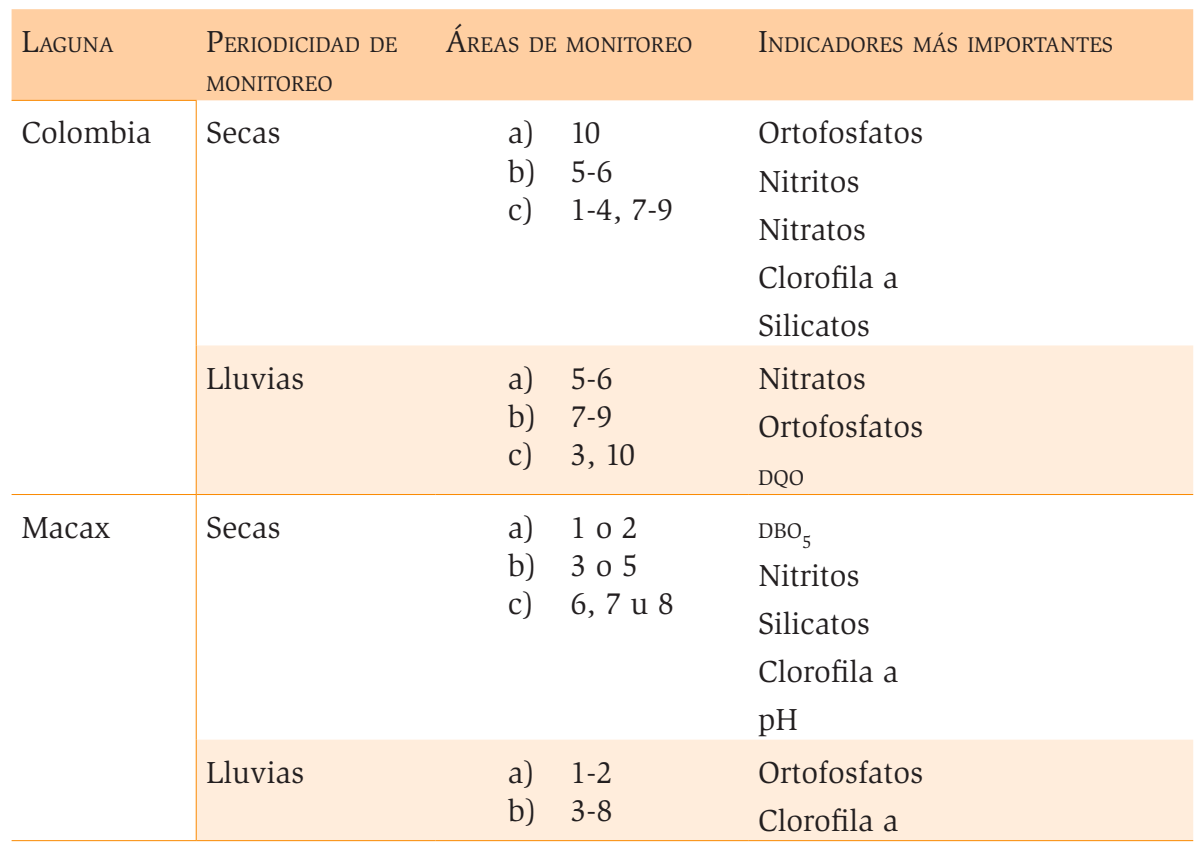

* Los números de los sitios de monitoreo corresponden a las estaciones de colecta mostradas en las figuras 1 y 2 .

En la laguna Colombia, por la dinámica del sistema, durante el tiempo de lluvias no fue posible establecer una diferenciación espacial tan clara como en el de secas. Debido a ello se sugiere que durante las lluvias se implementen tres áreas para el monitoreo de la calidad del agua: la primera y la segunda estarían comprendidas por las estaciones 5 a 9, que corresponden al este y oeste del sistema. El grupo formado por las estaciones 3 y 10 (sureste y norte de la laguna) se propone como la tercera área para el monitoreo de calidad del agua del sistema.

En cuanto a la laguna Macax, el cambio gradual en las variables físicas y químicas durante las secas obedece a la influencia de un canal amplio que comunica al sistema lagunar con el mar. Tal como se observa en la figura 2, la forma de esta laguna está definida por un eje principal con dirección surestenoroeste; el mencionado canal de comunicación tiene aproximadamente $600 \mathrm{~m}$ 
de longitud y entre 80 y $100 \mathrm{~m}$ de ancho. Por lo tanto, se recomienda que durante las secas se establezcan tres áreas de monitoreo de calidad del agua: las secciones sureste (estaciones 1 o 2 ), centro (estaciones 3 o 5 ) y noroeste del sistema (estaciones 6, 7 u 8).

Durante la temporada de lluvias, en la laguna Macax se encontraron discontinuidades en dos regiones debido a la concentración de ortofosfatos y de clorofila a. Por esto se sugiere establecer dos puntos de monitoreo de calidad del agua durante esta temporada: uno al extremo sureste (estaciones 1 o 2) y el segundo en cualquier punto desde el centro hacia el noroeste (estaciones 3 a 8).

Al observar el cuadro 6 se identifica la necesidad de considerar variables como la concentración de nitrógeno, fósforo y clorofila a en las campañas de monitoreo. Las tres variables, junto con el oxígeno disuelto en el agua, son los componentes principales del índice trófico TRIX. Es por ello que los resultados generados a partir de este índice trófico parecen ser más informativos en cuanto a la dinámica básica (espacial y temporal) de cada laguna. Por lo tanto, este estudio aporta evidencia empírica que sugiere considerar otros índices que tengan en cuenta las características intrínsecas de cada sistema (y sitios) para evaluar su comportamiento y proponer métodos adecuados para su manejo (Contreras-Espinosa, Castañeda-López y García-Nagaya, 1994; Carlson, 1997; Herrera-Silveira, 2006; Aranda Cirerol, 2004), sin circunscribirse exclusivamente a las normas (nacionales y extranjeras) de límites máximos permisibles.

Aunque el índice trófico mostró que el agua es de tipo oligotrófica, y pese a que de forma directa se observa con alta transparencia y aparentemente con posibilidades de uso turístico de contacto directo, en realidad se encontró que sobrepasa los límites máximos permisibles en la concentración de nutrimentos según los criterios ecológicos para la protección de la vida acuática marina. Además, su calidad puede clasificarse como pobre según los criterios de la EPA. Por ello no es recomendable usar estas lagunas para el uso recreativo de contacto directo ni para el consumo humano.

Con todo, dada la belleza escénica de los sitios y por ubicarse dentro o cerca de zonas sujetas a preservación o conservación ecológica (ahora clasificadas como sitios prioritarios para la conservación) (Conabio/Conanp/TNc/Pronatura, 2007), el aprovechamiento turístico sugerido es el de etnoturismo, senderismo, fotografía rural, pesca deportiva o pesca recreativa, actividades que podrían fortalecer el desarrollo del turismo de naturaleza en ambas lagunas. 


\section{Conclusiones}

Conforme aumenta el estudio de las zonas costeras en México es más evidente la necesidad de establecer, en el corto plazo, las características básicas de los sistemas lagunares costeros, así como comprender su dinámica particular (tanto espacial como temporalmente) para trabajar en la construcción de su inventario y monitoreo y en la generación de propuestas que permitan su manejo en función de sus atributos ambientales.

En el presente estudio se contrastan diversas fuentes que hacen posible definir cuál es la metodología que proporciona más información para delinear la dinámica básica de algunas lagunas costeras de Quintana Roo en cuanto a su calidad de agua y tener la posibilidad de generar propuestas de manejo.

De acuerdo con el análisis efectuado en las lagunas Colombia y Macax y siguiendo los Criterios Ecológicos de Calidad del Agua CE-CCA-001/89, la NOM-001ECOL-1996 y los criterios de calidad de aguas estuarinas y costeras de la EPA, el agua presenta cuatro características que según lo analizado en la sección previa y lo referente al WQI en el cuadro 5 pueden resumirse en lo siguiente:

1. Las lagunas sobrepasan los límites máximos permisibles en la concentración de nutrimentos, según los criterios ecológicos para la protección de la vida acuática marina

2. La calidad del agua puede clasificarse como pobre según los criterios de la EPA.

3. En apariencia, esta condición es estática (semejante) en la escala espacial.

4. No es recomendable el uso recreativo de contacto directo en estas lagunas.

Las herramientas estadísticas proporcionan información complementaria; por un lado, el análisis de los conglomerados de datos muestra que en ambas lagunas hay discontinuidades; esto es, hay diferencias espaciales dentro de ellas en función de las variables determinadas. Esto es relevante para tomar decisiones de manejo en cada laguna.

El ACP apunta a que algunas variables indicadoras adquieren más importancia que otras, dependiendo de la estación climática. Esto significa que el moni- 
toreo no debe ajustarse específicamente a un número limitado de variables. Se recomienda el aprovechamiento de los sistemas analizados a través de una variante del turismo de naturaleza, pero no el uso recreativo de contacto directo.

\section{Agradecimientos}

El proyecto se desarrolló con apoyo del Fondo Mixto Conacyt-Gobierno del Estado de Quintana Roo, registro Fomix-123254. Jorge Sulub Tolosa e Irma Ortíz-Borge fueron tesistas del proyecto. La Fundación de Parques y Museos de Cozumel y la Secretaría de Marina en Isla Mujeres otorgaron facilidades de acceso a los sistemas estudiados. La División de Desarrollo Sustentable de la Universidad de Quintana Roo apoyó la realización de este proyecto, dentro del cuerpo académico Vulnerabilidad y Biodiversidad de Sistemas Acuáticos Continentales y Costeros.

\section{Fuentes consultadas}

Aguilar, V. (2003). "Aguas continentales y diversidad biológica de México: un recuento actual". Biodiversitas, 8 (48), mayo, 1-15.

APHA (1986). Standard Methods for the Examination of Water and Wastewater. 16a ed. Washington: American Public Health Association. 1527 pp.

Aranda Cirerol, N. (2004). "Eutrofización y calidad del agua en una zona costera tropical”. Tesis de doctorado. Barcelona: Universidad de Barcelona. $229 \mathrm{pp}$.

Azuz-Adeath, I. y E. Rivera-Arriaga (2004). "Escalas espaciales y temporales del manejo costero”, en E. Rivera-Arriaga et al. (eds.). El manejo costero en México. Campeche: Universidad Autónoma de Campeche/Secretaría de Medio Ambiente y Recursos Naturales (Semarnat)/Centro de Enseñanza Técnica y Superior (CETYs)/Universidad de Quintana Roo (UQROO), 27-37.

Carlson, R.E. (1997). “A Trophic Index for Lakes”. Limnology and Oceanography, 22, 361-369.

Conabio/Conanp/Tnc/Pronatura (2007). Análisis de vacíos y omisiones en conservación de la biodiversidad marina de México: océanos, costas e islas. 
México: Comisión Nacional para el Conocimiento y Uso de la Biodiversidad/Comisión Nacional de Áreas Naturales Protegidas/The Nature Conservancy-Programa México/Pronatura. 128 pp.

Contreras-Espinosa, F., O. Castañeda-López y A. García-Nagaya (1994). “La clorofila a como base para un índice trófico en lagunas costeras mexicanas”. Anales del Instituto de Ciencias del Mar y Limnología, 21, 55-66. DGN-SE (2001a). “NMX-AA-028-SCFr-2001. Análisis de agua -determinación de la demanda bioquímica de oxígeno en aguas naturales, residuales $\left(\mathrm{DBO}_{5}\right)$ y residuales tratadas- Método de prueba (cancela a la NMX-AA-028-1981)”. México: Dirección General de Normas-Secretaría de Economía.

------ (2001b). “NMX-AA-030-scFi-2001. Análisis de agua -determinación de la demanda química de oxígeno en aguas naturales, residuales y residuales tratadas- Método de prueba (cancela a la NMX-AA-030-1981)”. México: Dirección General de Normas-Secretaría de Economía.

Environmental Protection Agency. 2004. National Condition report II. Office of Water. EPA-620-R03/0002,

Herrera-Silveira, J.A. (2006). "Lagunas costeras de Yucatán (SE, México): Investigación, diagnóstico y manejo”. Ecotrópicos, 19 (2), 94-108.

Herrera-Silveira, J.A., F.A. Comín y L. Capurro (2004). "Los usos y abusos de la zona costera en la Península de Yucatán”, en E. Rivera-Arriaga et al. (eds.). El manejo costero en México. Campeche: Universidad Autónoma de Campeche/Semarnat/CETYs/uQRoo, 387-396.

Herrera-Silveira, J.A., J. Ramírez-Ramírez y J. Zaldivar (1998). “Overview and Characterization of the Hydrology and Primary Producer Communities of Selected Coastal Lagoons of Yucatan, Mexico". Aquatic Ecosystem Health and Management Society, 1, 353-372.

Parsons, T.R., Y. Maita y C.M. Lalli (1984). A Manual of Chemical and Biological Methods for Seawater Analysis. Oxford: Pergamon Press.

Reyes, E. y M. Merino (1991). "Diel Dissolved Oxygen Dynamics and Eutrophication in a Shallow, Well-Mixed Tropical Lagoon (Cancun, Mexico)". Estuaries, 14 (4), 372-381.

Sandoval-Rojo, L.C. et al. (1988). "Phytoplankton Productivity in the Barra de Navidad Coastal Lagoon on the Pacific Coast of Mexico”. Revue d'Hydrobiologie Tropicale, 21, 101-108.

Secretaría de Desarrollo Urbano y Ecología (1989). Acuerdo por el que se esta- 
blecen los Criterios Ecológicos de Calidad del Agua CE-CCA-001/89. Diario Oficial de la Federación (en línea). Disponible en: http://dof.gob. $\mathrm{mx} /$ nota_detalle.php? codigo $=4837548 \&$ fecha $=13 / 12 / 1989$ [2012, 12 de julio].

Secretaría de Medio Ambiente y Recursos Naturales (1997). "Norma Oficial Mexicana NOM-001-ECoL-1996, que establece los límites máximos permisibles de contaminantes en las descargas de aguas residuales en aguas y bienes nacionales”. Diario Oficial de la Federación [en línea], 6 de enero. Disponible en: http://www.profepa.gob.mx/innovaportal/ file/3290/1/nom-001-ecol-1996.pdf

Tran, K.C. et al. (2002). "Status of Water Quality at Holbox Island, Quintana Roo State, Mexico". Aquatic Ecosystems Health \& Management, 5, 173-189. Vollenweider, R.A. et al. (1998). "Characterization of the Trophic Conditions of Marine Coastal Waters with Special Reference to the NW Adriatic Sea: Proposal for a Trophic Scale, Turbidity and Generalized Water Quality Index”. Environmetrics, 9, 329-357. 